\title{
Morphology and one immunohistochemical marker are enough for diagnosis of primary thyroid angiosarcoma
}

\author{
Ion Negurăa, ${ }^{*}$, Minerva Codruța Bădescu ${ }^{2,3}$, Ciprian Rezușa,3, Radu Dănilă4, \\ Alexandru Florin Florescu ${ }^{5}$, Mihaela Blaj6, Eugenia Moroșan7, Delia Gabriela \\ Ciobanu Apostol ${ }^{1,7}$
}

${ }^{1}$ Pathology Department, "Sf. Spiridon" Emergency County Clinical Hospital, Iasi, Romania; 2Department of Internal Medicine, "Grigore T. Popa" University of Medicine and Pharmacy, lasi, Romania; ${ }^{3} \mid I^{\text {rd }}$ Internal Medicine Clinic, "Sf. Spiridon" Emergency County Clinical Hospital, lasi, Romania; ${ }^{4} 3^{\text {rd }}$ Surgical Department, "Sf. Spiridon" Emergency County Clinical Hospital, lasi, Romania; ${ }^{5}$ Endocrinology Department, "Sf. Spiridon" Emergency County Clinical Hospital, lasi, Romania; ${ }^{6}$ Department of Anesthesia and Intensive Care, "Sf. Spiridon" Emergency County Clinical Hospital, lasi, Romania; "Pathology Department, "Grigore T. Popa" University of Medicine and Pharmacy, lasi, Romania

\begin{abstract}
Primary thyroid angiosarcoma is a very rare and extremely aggressive mesenchymal malignant neoplasm showing morphological and immunophenotypic evidence of endothelial cell differentiation. Early diagnosis of this tumor along with radical thyroidectomy followed by postoperative radiotherapy and chemotherapy are essential for adequate management of the patient. Currently available data on diagnosis and treatment options of this neoplasm are limited because it is a rare disease in endocrine organs. Raising awareness regarding its diagnosis can help to optimize the treatment and to improve the survival of the patient. We present the case of a 72-year-old female patient with multiple comorbidities who addressed to the hospital with obstructive respiratory symptoms: dyspnea, wheezing and hoarseness. The investigations, both clinical and paraclinical, identified a local invasive cervical mass located mainly in the left thyroid lobe, whose immunohistochemical examination confirmed primary thyroid angiosarcoma. Although this type of neoplasm is described mainly in the Alpine regions, it can appear in lower altitude regions and this tumor needs to be differentiated from a highgrade neoplasm (anaplastic thyroid carcinoma).
\end{abstract}

Keywords: thyroid primary angiosarcoma; epithelioid angiosarcoma; mesenchymal neoplasm; immunohistochemistry; CD31

\section{Introduction}

Angiosarcoma is a malignant mesenchymal tumor that shows morphological and immunohistochemical aspects of endothelial cell differentiation [1]. Soft tissue sarcomas represent a category of malignant

Received: April 2021; Accepted after review: June 2021; Published: June 2021.

${ }^{\star}$ Corresponding author: Ion Negura, Pathology Department, "Sf. Spiridon" Emergency County Clinical Hospital, 1 Independentei Bvd, Iasi, Romania

Email: dr.neguraionut@gmail.com tumors, of which $2-4 \%$ are angiosarcomas, mainly encountered in adults and the elderly [2]. Mesenchymal tumors are rare in thyroid, primary thyroid angiosarcoma being the most frequent one [3]. Beside iodine deficiency, irradiation for other malignancies or radiation exposure at work and professional exposure to vinyl chloride predispose to thyroid angiosarcoma $[5,6]$. Although thyroid angiosarcoma is considered a geographically restricted type of cancer, such tumors were reported outside of Alpine areas, in seaside areas or even in flatlands [7]. Adult females in 
the seventh decade of life have the highest risk of developing this type of tumor, the risk being 4.5 times higher in women compared to men $[8,9]$. Primary thyroid angiosarcoma is an aggressive malignancy with a very poor prognosis. The patient usually has a mean survival of 6 months and dies from metastatic disease. Most common organs involved in the metastatic process are: bones, lungs, lymph nodes and soft tissues [10]. Thyroid angiosarcoma has variable and usually nonspecific clinical signs and symptoms which depend on: the size of the tumor, position, extension outside the gland, presence or absence and localization of metastases. Generally, angiosarcoma of the thyroid presents with symptoms of obstruction because it has an accelerated growth rate. These pressure symptoms include dysphagia, hoarseness and shortness of breath and are the result of compression or even infiltration of neck nerves and cervical aero-digestive structures. Cervical pain is another way in which this tumor can manifest. Other manifestations which are paraneoplastic signs and symptoms can include: anemia, fever and leukocytosis. When metastases are present, the clinical manifestations vary depending on the affected organ [11]. Here we report a case of primary angiosarcoma of the thyroid diagnosed in a 72-year-old female patient with multiple comorbidities and a personal history of goiter, from a non-Alpine area.

\section{Case presentation}

A 72-year-old female patient with multiple comorbidities: type 2 diabetes mellitus, hypertensive and ischemic dilated cardiomyopathy, congestive heart failure, stage $3 b$ chronic kidney disease, recent SARS CoV2 infection presented at the emergency room with a 2-month history of obstructive respiratory symptoms, with progressive aggravation. Prior to that the patient was addressed to the surgery department by an endocrinologist, but due to the SARS CoV2 infection thyroidectomy was postponed. The patient was from a mildly iodine insufficient non-Alpine area, yet the iodine status of the patient was not available. Clinical examination of the anterior cervical region and paraclinical investigations (cervical computed tomography) identified a 93/90/76 mm large, inhomogeneous soft consistency mass on the topography of the left thyroid lobe that displaced to the right the trachea and cervical esophagus. Otorhinolaryngology examination identified left recurrent laryngeal nerve paralysis. There was no evidence of other primary malignancies or metastatic dissemination at the thoracic and cervical CT. Blood examination showed euthyroid status, normal calcitonin level, moderate normochromic and normocytic anemia, and a stage $3 b$ chronic kidney disease. Mild hypocalcemia was also present. Despite age and significant comorbidities resulting in high anesthesia risk, intense obstructive respiratory symptoms established the indication for surgery. Intraoperative examination revealed a solid tumor mass on the topography of the left thyroid lobe with invasion of infrahyoidian muscles, trachea, left recurrent laryngeal nerve and cervical esophagus. Lymphadenopathy was not identified in the central and lateral compartments. A total thyroidectomy and excision of the invaded infrahyoid muscles was performed. Postoperatively, following a period of 48 hours when the patient was maintained intubated and ventilated, a major improvement of the respiratory status was noticed. The surgical specimen was fixed in $10 \%$ neutral buffered formalin solution. At gross examination, a tumor that measured $80 / 60 / 40 \mathrm{~mm}$, partially circumscribed by a pseudocapsule, was observed. The cut surface was variegated with solid and cystic areas. Necrosis was present in the solid areas and hemorrhage was extensive. Both lobes of the thyroid gland were involved by the tumor along with the isthmus and the infrahyoid muscles (Figure 1). 

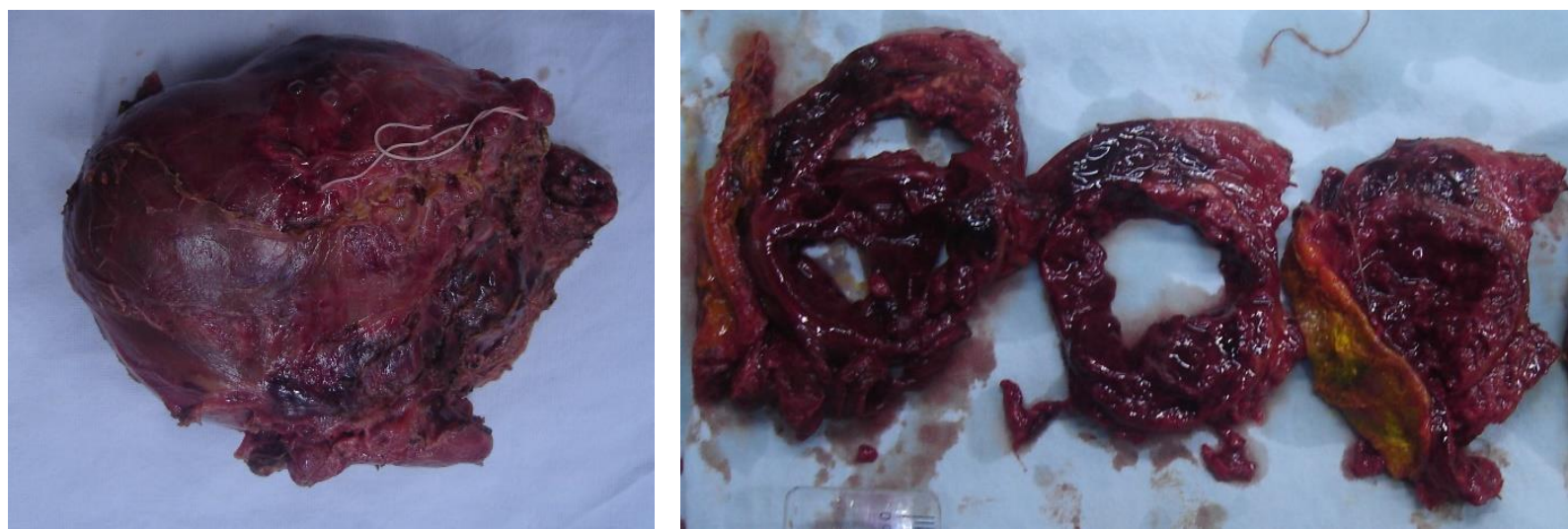

Fig. 1. (A) Gross thyroidectomy specimen including the tumor mass in the left lobe (prior to sectioning). (B) Cut surface of the tumor with solid necrotic areas, cystic degeneration and extensive hemorrhage. The resected infrahyoid muscles are also present (yellow paint).

Microscopic examination identified a vasoformative high grade proliferation with large uni- or multinucleated epithelioid cells with prominent eosinophilic nucleoli and abundant eosinophilic cytoplasm lining abnormal vascular channels containing papillary fronds (Figure 2A) or growing in solid sheets (Figure 2B). Intracytoplasmic lumina with erythrocytes were present in some of the cells. Extensive areas of hemorrhage and tumor necrosis were also noted. The mitotic rate was high with frequent atypical mitotic figures. Perineural invasion and angioinvasion were present. Extra-thyroid soft tissues including the infrahyoid muscles were infiltrated by the tumor. The residual thyroid gland showed features of multinodular colloid goiter.
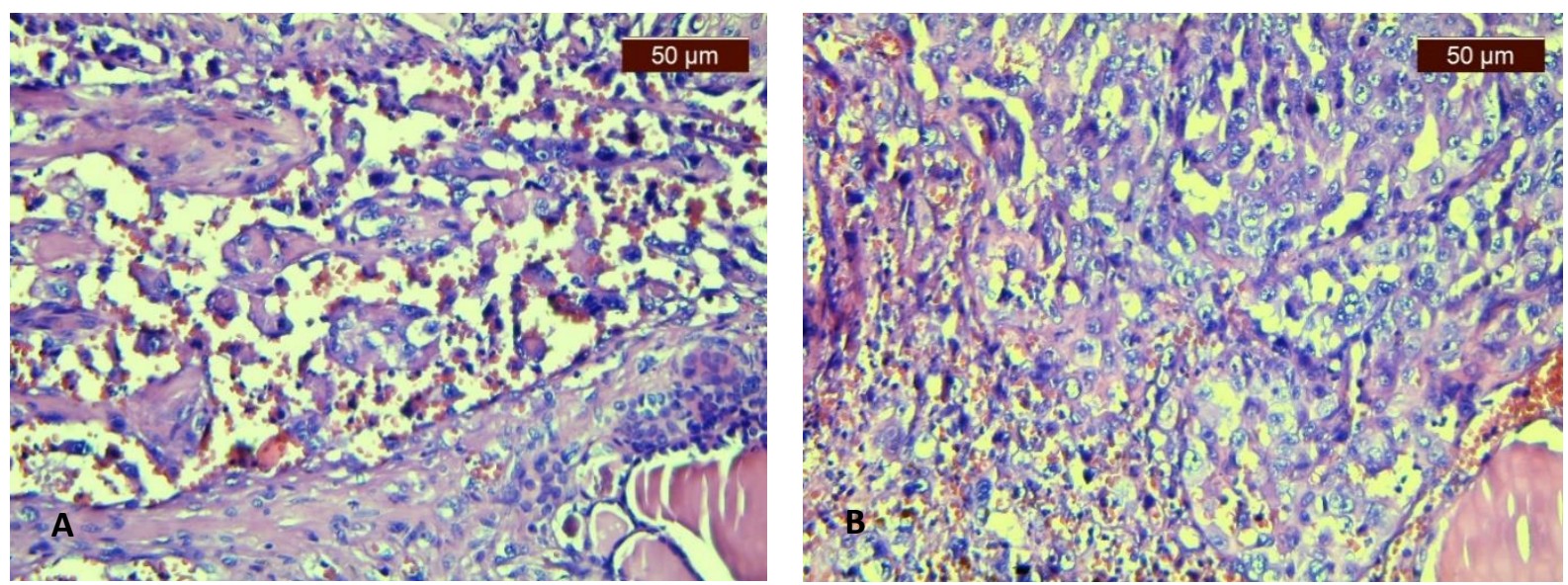

Fig. 2. Microscopical aspect of thyroid angiosarcoma. Vasoformative area of the tumor with large uni- or multinucleated epithelioid tumor cells lining abnormal vascular channels containing papillary fronds $(A)$ or in solid sheets (B). (HE, x200)

On immunohistochemistry, among endothelial cell markers, only CD31 showed intense diffuse membrane positivity (Figure 3A). Other two endothelial cell markers (CD34 and D2-40) were negative (Figures $3 B$ and $3 C)$. CK AE1/AE3, an epithelial differentiation marker, was focally positive (Figure 3D). TTF1 , thyroglobulin and calcitonin were diffusely negative in tumor cells. Based on the morphological aspects and immunohistochemical profile, the final diagnosis was primary angiosarcoma of thyroid with extra-thyroid extension to infrahyoid muscles. Postoperatively, the patient developed hypoparathyroidism with severe hypocalcemia that responded to intravenous calcium administration and oral alfacalcidolum (activated vitamin D3). The patient was discharged in good clinical condition with recommendation for clinical and paraclinical endocrinological evaluation and initiation of hormone replacement therapy. 
After discharge, the patient did not receive postoperative chemotherapy or radiotherapy due to chronic kidney disease and died one month later in the nephrology unit.

\section{Discussion}

Primary angiosarcoma of the thyroid is a recent tumor type recognized in 2013 by WHO [12]. Previously, this tumor was considered a vascular type of anaplastic thyroid carcinoma and not a malignant mesenchymal tumor with endothelial cell differentiation. Only when immunohistochemistry demonstrated the endothelial differentiation of the tumor in 1986 this entity started to be recognized [13]. Angiosarcoma is a rare aggressive tumor with mesenchymal origin, the main risk factors being irradiation and chronic lymphedema.
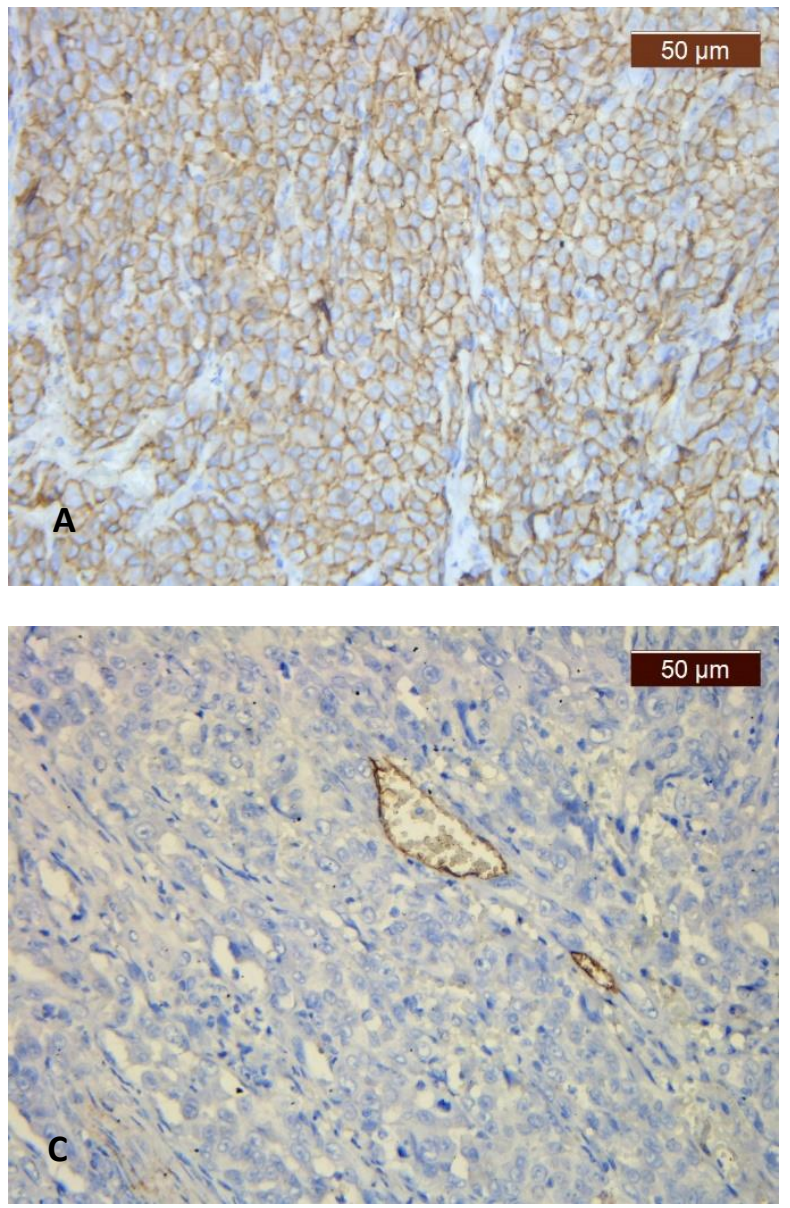

The most frequent locations are soft tissues and skin but cases involving spleen, breast, liver and bone have been reported. This tumor has poor prognosis mainly because of incomplete resection which predisposes to recurrence and because metastases develop early in the course of the disease [14].

Primary angiosarcoma of thyroid gland is a very rare type of cancer and until 2020 there were only 59 histologically confirmed cases reported in the English literature [10]. In this paper we report the case of a female patient from a non-Alpine area (Galati, Romania) that was diagnosed with primary thyroid angiosarcoma. To the best of our knowledge, this is the third case reported in Romania [4, 24]. The data available so far indicate a peak incidence in the seventh decade of life, with a female predominance, which is also confirmed by our case [10].
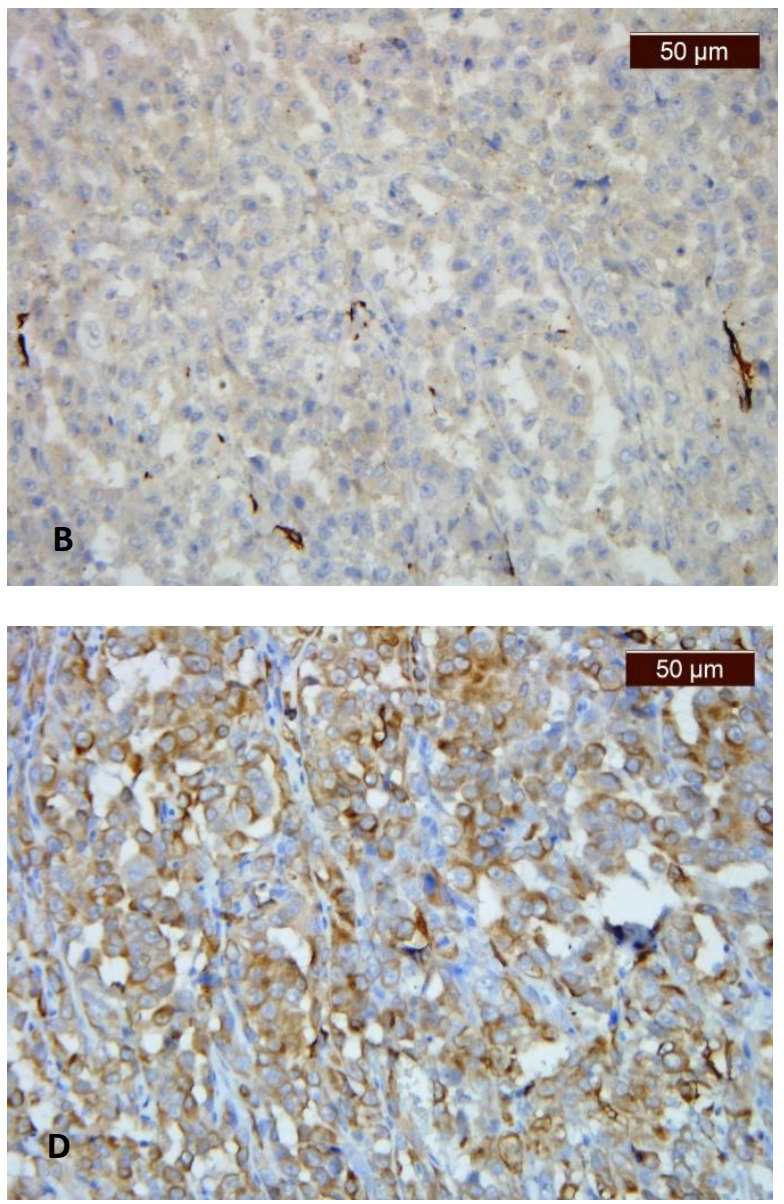

Fig. 2. Immunohistochemical profile of thyroid angiosarcoma. A: Strong and diffuse membrane positivity for CD31 in solid areas (IHC, anti-CD31 antibody, x200); B: Total negativity for CD34 (IHC, anti-CD34 antibody, x200); C: Total negativity for D2-40 (IHC, anti-D2-40 antibody, x200); D: Positivity for CKAE1/AE3 in vascular channels (IHC, anti-CK AE1/AE3 antibody, x200). 
lodine deficiency and goiter are closely related [15]. In Romania, Moldova is a region with iodine deficiency and with endemic thyroid goiter that predisposes to this type of thyroid malignancy [4]. The main diagnostic technique used to investigate thyroid nodules is fine needle aspiration biopsy. Surgical management of the patients with thyroid nodules is dependent on the diagnosis obtained after fine needle aspiration biopsy of the lesion [16]. In this case, due to the fast growing tumor, the cardiovascular status and the anticoagulant and antiplatelet therapy, the endocrinologist referred the patient directly to surgery. Due to the severe respiratory impairment at hospital admission, the patient was directly evaluated by computed tomography, both for diagnostic purposes and to establish the appropriate therapeutic protocol. Also, particularly for our case was the normal thyroid function in the conditions of the presence of a large tumor mass. A total thyroidectomy without lymphadenectomy and excision of the infrahyoid muscles with tumor invasion was performed.

Thyroid angiosarcomas measure between 3 and $10 \mathrm{~cm}$ in size and appear as circumscribed pseudoencapsulated lesions, but are invasive tumors on microscopic examination. On cut surface, the appearance is variegated, with solid and cystic zones. The solid areas are necrotic and hemorrhage is frequently encountered [17]. Microscopically, angiosarcomas of the thyroid are similar to deep soft tissue angiosarcomas [18]. Viable tumor cells are usually found at the periphery of the lesion because the central portion of the tumor shows extensive necrosis and hemorrhage admixed with dense inflammation and fibrosis. The vascular nature of the tumor is manifested by presence of anastomosing vascular channels that contain papillary fronds which are lined by atypical tumor cells. In poorly differentiated areas a solid, sheet-like growth pattern is present which can overlap with the morphology of anaplastic thyroid carcinoma. Primary thyroid angiosarcomas frequently present epithelioid cytological morphology $[9,12,19]$. The nuclei of the tumor cells are round, vesicular or hyperchromatic with prominent nuclear membranes and evident eosinophilic nucleolus. Cytoplasm is abundant and eosinophilic [20]. Sometimes the neoplastic cells can be bi- and/or multinucleated. Angiosarcoma is usually very mitotically active with frequent atypical mitotic figures. CD31 (membranar) and erythroblast transformation specific related gene (ERG) (nuclear) are the most specific and sensitive positive markers that help diagnose angiosarcoma. CD34 and factor VIII-related antigen are two additional endothelial cell markers with variable expression in angiosarcomas [21]. In our case, only CD31 was positive, while CD34 was negative. AntiD2-40 antibody, another endothelial cell marker that is more specific for lymphatic endothelium was negative in the tumor cells. Epithelioid subtypes of angiosarcomas are frequently positive for keratin and EMA and can be falsely diagnosed as carcinomas [18]. CK AE1/AE3 was positive in our case and based on the morphology and patient age, anaplastic thyroid carcinoma was considered as a potential differential diagnosis, but the presence of anastomosing vascular channels lined by malignant tumor cells and CD31 positivity along with the fact that anaplastic thyroid carcinoma is always CD31 negative were enough for the positive diagnosis of primary thyroid angiosarcoma. PAX8, a marker that is frequently positive in anaplastic thyroid carcinoma is not available in our institution. We performed a series of other markers (TTF-1, thyroglobulin, calcitonin) that are frequently used for differential diagnosis of thyroid tumors (primary or metastatic) and all were negative [22].

The ultrasound examination in primary thyroid angiosarcoma shows inhomogeneously hypoechoic lesions with indistinct margins that contain hyperechoic zones. Computed tomography imaging frequently shows inhomogeneous lesions predominantly hypodense with hyperdense zones. Inhomogeneous hypo- to hyperintense lesions are frequently described on magnetic resonance imaging [23]. The cervico-thoracic computed tomography showed a 93/90/76 mm large, inhomogeneous soft consistency mass on the topography of the left thyroid lobe that displaced to the right the trachea and the cervical esophagus. 
Extra-thyroidal invasion was present on microscopic examination. Lymph nodes were not resected, but angioinvasion was identified. According to data from a series of three cases and literature review angioinvasion may be a possible risk factor for disease progression [24]. In a larger series of cases [25], extrathyroidal invasion was identified as a deleterious prognostic factor. Due to its rarity, the treatment of primary thyroid angiosarcoma is not standardized and no "gold standard" exists. Radical surgery along with postoperative radiotherapy improves the prognosis and the overall survival, and currently it is the best therapeutic strategy [26]. The role of chemotherapy in primary thyroid angiosarcoma has not been established yet [27]. Although several attempts have been made with ifosfamide, epirubicin, taxanes and doxorubicin, either as monotherapy or in combination, the overall outcome was still poor [11]. Since in primary thyroid angiosarcoma the cell of origin is a mesenchymal thyroid cell, this tumor does not respond to radioactive iodine therapy. One study investigated the role of anti-VEGF agents in the treatment of this tumor but the results were not satisfactory [28]. Further studies are needed to confirm and verify the positive effects of these new drugs on thyroid angiosarcoma.

\section{Conclusion}

Primary thyroid angiosarcoma can morphologically mimic anaplastic thyroid carcinoma which is a more frequent type of thyroid malignancy. Distinction between these two types of thyroid tumors is very important because the prognosis and therapy are different. Considering the vascular pattern of angiosarcoma and the higher sensibility and specificity of CD31 compared to CD34, we highlight that CD31 is essential when designing the panel for immunohistochemical tests. Raising awareness to pathologists and clinicians about this type of thyroid tumor is very important because is a rare tumor, especially in non-Alpine regions and sometimes is hard to distinguish it from other thyroid tumors. For both clinicians and the patient the correct diagnosis is important in order to increase the chances of survival by early diagnosis and appropriate treatment. It is also necessary to record new cases of this rare type of thyroid tumor in order to contribute with supplementary data on diagnosis, therapeutic approach, prognosis and final outcome of the patient.

\section{Consent}

Written informed consent was obtained from the patient for publication of this case report.

\section{Competing interests}

The authors declare that they have no competing interests.

\section{References}

1. Young R, Brown N, Reed M, Hughes D, Woll P. Angiosarcoma. Lancet Oncol 2010; 11(10):983991.

2. Toro JR, Travis LB, Wu HJ, et al. Incidence patterns of soft tissue sarcomas, regardless of primary site, in the surveillance, epidemiology and end results program, 1978-2001: an analysis of 26,758 cases. Int $J$ Cancer 2006; 119(12):2922-2930.

3. Surov A, Gottschling S, Wienke A, Meyer $\mathrm{HJ}$, Spielmann RP, Dralle $H$. Primary thyroid sarcoma: a systematic review. Anticancer Res 2015; 35:5185-5191.

4. Halalau F, Laky D. Hemangiosarcoma of the thyroid gland. Histological diagnosis problems. Morphol Embryol (Bucur) 1983; 29(3):195-196.

5. Collini $P$, Barisella $M$, Renne $S L$, et al. Epithelioid angiosarcoma of the thyroid gland

without distant metastases at diagnosis: report of six cases with a long follow-up. Virchows Arch 2016; 469:223-232.

6. Marina M, Corcione L, Serra MF, Ferri T, Silini EM, Ceresini G. Primary epithelioid angiosarcoma of the thyroid in a patient occupationally exposed to radiations. Front Endocrinol 2018; 9:577. doi:10.3389/fendo.2018.00577.

7. Ortiz J, Villabona C, Bengoechea 0 , et al. Primary epithelioid angiosarcoma of the thyroid: an infrequent malignant thyroid tumor. Endocrinol Nutr 2008; 55:181-183.

8. Rosai J, Carcangiu ML, DeLellis RA. Sarcomas. In: Atlas of Tumor Pathology. Tumors of the Thyroid Gland, 3rd series. Washington, DC: Armed Forces Institute of Pathology 1992. 
9. Papotti M, Arrondini M, Tavaglione V, Veltri A, Volante M. Diagnostic controversies in vascular proliferations of the thyroid gland. Endocr Pathol 2008; 19:175-183.

10. Huang SH, Wu SC. Primary angiosarcoma of the thyroid in an Asian woman: a case report with review of the literature. Case Rep Pathol 2020; https://doi.org/10.1155/2020/90685 06.eCollection.

11. De Felice F, Moscatelli E, Orelli S, Bulzonetti $\mathrm{N}$, Musio D, Tombolini V. Primary thyroid angiosarcoma: a systematic review. Oral Oncol 2018; 82:48-52.

12. Jo VY, Fletcher CD. WHO classification of soft tissue tumors: an update based on 2013 (4th) edition. Pathology 2014; 46:95-104.

13. Chan YF, Ma L, Boey JH, Yeung HY. Angiosarcoma of the thyroid. An immunohistochemical and ultrastructural study of a case in a Chinese patient. Cancer 1986; 57:2381-2388.

14. Buehler D, Rice SR, Moody JS, et al. Angiosarcoma outcomes and prognostic factors: a 25-year single institution experience. Am J Clin Oncol 2014; 37(5):473-479.

15. Asmelash D, Tesfa K, Biadgo B. Thyroid dysfunction and cytological patterns among patients requested for thyroid function test in an endemic goiter area of Gondar, North West Ethiopia. Int J Endocrinol 2019; 2019:91067679106767.

16. Feldkamp J, Führer D, Luster M, Musholt TJ, Spitzweg C, Schott M. Fine needle aspiration in the investigation of thyroid nodules. Dtsch Arztebl Int 2016; 113(20):353-359.

17. Maiorana A, Collina G, Cesinaro AM, et al. Epithelioid angiosarcoma of the thyroid. Clinicopathological analysis of seven cases from non-Alpine areas. Virchows Arch 1996; 429:131-137. PMID:8917714

18. Fletcher CD, Beham A, Bekir $S$, et al. Epithelioid angiosarcoma of deep soft tissue: a distinctive tumor readily mistaken for an epithelial neoplasm. Am J Surg Pathol 1991; 15(10):915-24. PMID:1718176.

19. Eusebi V, Carcangiu ML, Dina R, et al. Keratinpositive epithelioid angiosarcoma of thyroid. A report of four cases. Am J Surg Pathol 1990; 14:737-47. PMID:1 696070

20. Ryska A, Ludvikova M, Szepe $P$, et al. Epithelioid haemangiosarcoma of the thyroid gland. Report of six cases from a non-Alpine region. Histopathology 2004; 44:40-8. PMID:14717668

21. Miettinen $M$, Lindenmayer $A E$, Chaubal $A$. Endothelial cell markers CD31, CD34, and BNH9 antibody to $\mathrm{H}$ - and $\mathrm{Y}$-antigensevaluation of their specificity and sensitivity in the diagnosis of vascular tumors and comparison with von Willebrand factor. Mod Pathol 1994; 7(1):82-90. PMID:7512718

22. Buzdugă $C M$, Costea $C F$, Cărăuleanu $A$, et. al. Protean, cytologycal, histological and immunohistochemical appearances of medullary thyroid carcinom: curent undates. Rom J Morphol Embryol 2019; 60(2):369-381

23. Surov A, Holzhausen HJ, Machens A, Dralle H. Imaging findings of thyroidal sarcoma. Clin Imaging 2014; 38:826-830.

24. Nechifor-Boila A, Decaussin-Petrucci M, Vargallyés A, Chinezu L, Carasca C, Borda A. Angioinvasion as a factor for predicting aggressive outcome in primary thyroid angiosarcoma: three case reports and literature review. Pol J Pathol 2018; 69:53-61. doi: 10.5114/pjp.2018.75337

25. Wick MR, Eusebi V, Lamovec J, Ryska A. Angiosarcoma. In: Lloyd RV,Osamura RY, Kloppel G, Rosai J, editors. WHO Classification of Tumors of Endocrine Organs, 4th edn. Lyon. WHO Press (2017). p 129-32.

26. Mattavelli D, Miceli R, Radaelli $S$, et al. Head and neck soft tissue sarcomas: prognostic factors and outcome in a series of patients treated at a single institution. Ann Oncol 2013; 24:2181-2189.

27. Abraham JA, Hornicek FJ, Kaufman AM, et al. Treatment and outcome of 82 patients with angiosarcoma. Ann Surg Oncol 2007; 14:19531967.

28. Park MS, Ravi V, Araujo DM. Inhibiting the VEGF-VEGFR pathway in angiosarcoma, epithelioid hemangioendothelioma, and hemangiopericytoma solitary fibrous tumor. Curr Opin Oncol 2010; 22:351-355. 